\title{
Natural versus synthetic fixative in oral cytological smears - A double blind study
}

\author{
Savitri M Nerune ${ }^{1, *}$, Mahmood Nawaz Khan², R.M Potekar ${ }^{3}$, Vijayalaxmi Patil ${ }^{4}$ \\ ${ }^{\mathbf{1}}$ Assistant Professor, ${ }^{2}$ Post Graduate, ${ }^{3}$ Professor, ${ }^{4}$ Assistant Professor, Dept. of Pathology, Shri B.M Patil Medical College, \\ Hospital and Research Centre, BLDE University, Vijayapura, Karnataka, India
}

\author{
*Corresponding Author: Savitri M Nerune \\ Email: nervunesavitri.mallikarjun@bldeuniversity.ac.in
}

Received: $5^{\text {th }}$ April, 2018

Accepted: $24^{\text {th }}$ April, 2018

\begin{abstract}
Objective: Fixation in cytology is to preserve and maintain clear and consistent morphological features. Ethanol is most commonly used a gold standard fixative in cytology, but it has few disadvantages such as expensive, flammable, evaporates easily, subjected to pilferage and not easily available. So honey can be used as fixative as it contains several minerals, trace elements, vitamins, carbohydrates and acts as a fixative because of its inherent properties by enabling cellular preservation and dehydration. Considering this objective of present study is to evaluate the fixative properties of honey in comparison with ethanol in a double blinded manner.

Materials and Methods: Two oral smears were obtained from each individual $(n=50)$ by gently scraping the buccal mucosa. One slide was fixed in ethanol $(95 \%)$ and the other in honey (20\% processed) for a minimum of 15 minutes and stained with Papanicolaou stain. Two separate pathologists who were blinded for the fixative used evaluated the slides for 5 parameters (Cell morphology, Nuclear and Cytoplasmic staining, Clarity and uniformity of staining,)

Results: Among 50 cases evaluated by both pathologists, results showed that honey fixed smears had acceptable nuclear and cytoplasmic staining, well preserved cell morphology, clarity and uniformity of staining as comparable to ethanol with no statistical difference between both fixatives.

Conclusion: Hence in search of a better, eco-friendly and cost effective fixative, $20 \%$ processed honey can be efficiently used in cytological smear fixation for the preservation of cellular details.
\end{abstract}

Keywords: Buccal smear, Ethanol, Fixation.

\section{Introduction}

Cytology provides rapid diagnosis by minimally invasive technique. Exfoliative cytology as a method of diagnosis was first introduced by Papanicolaou in 1943. Exfoliative cells from oral epithelium have been widely used in cytology to detect abnormal nuclear and cellular morphology depicting precancerous and cancerous changes. Buccal mucosa due to more surface area is widely affected when exposed to insults in oral cavity resulting in epithelial changes. ${ }^{1}$ Exfoliative oral cytological smears can play an important role in diagnosing lesions which are clinically not obvious or suspicious for malignancy and might obviate the need of invasive biopsy procedure. ${ }^{2}$

Accuracy of cytopathological diagnosis depends on multiple steps such as collection, proper fixation, staining and quality control. Among this fixation plays an important role in fixing the cells and, making them amenable to evaluation and reach an appropriate diagnosis. Ethanol is well known gold standard fixative used in cytology. Though it provides excellent preservation of cellular morphology certain limitations such as being not freely available, inflammable and being subjected to pilferage it loses its title from being called as an ideal fixative.

Therefore in search of an ideal fixative and to overcome limitations of ethanol, natural sweeteners especially honey are being extensively evaluated to study their inherent properties which can be exploited for tissue fixation. Honey due to its low $\mathrm{pH}$ and several enzymes and antibacterial properties due to presence of hydrogen peroxide can be introduced in cytology as natural fixative, which is eco-friendly and cheap. ${ }^{3,4}$

\section{Materials and Methods}

A cross sectional comparative study was carried out on healthy MBBS students Study period: $1^{\text {st }}$ June to $30^{\text {th }}$ June 2017.

After taking informed consent from the students two oral smears were obtained from each individual (n $=50$ ) by gently scraping the buccal mucosa. One slide was fixed in ethanol (95\%) and the other in honey $(20 \%$ processed) for a minimum of 15 minutes and stained with Papanicolaou stain. Two separate pathologists who were blinded for the fixative used evaluated the slides for 5 parameters (Cell morphology, Nuclear and Cytoplasmic staining, Clarity and uniformity of staining,) (Table 1)

Inclusion Criteria: All apparently healthy MBBS students were included in the study.

\section{Exclusion Criteria: Nil}

\section{Statistical Analysis}

Data was analyzed using Mean +/- S.D, Diagrams, Chi square test and $\mathrm{P}$ value of $<0.05$ was considered as significant. 


\section{Results}

Out of 50 cases, 28 were females and 22 were males. Out of 50 cases evaluated by first reviewer $100 \%$ of honey fixed (HF) slides and $98 \%$ of ethanol fixed (EF) showed better nuclear staining, (Fig 1a \&1b) whereas $92 \%$ of $\mathrm{HF}$ slides and $76 \%$ of $\mathrm{EF}$ slides showed acceptable nuclear staining by a second reviewer $\left(\mathrm{P}_{1}=0.315, \mathrm{P}_{2}=0.118\right)$. (Table 2 , Table 3 )

On analysis for cytoplasmic staining, by first reviewer $80 \%$ of honey fixed (HF) slides and $92 \%$ of ethanol fixed (EF),(fig 1a \&1b) were acceptable were as $88 \%$ of $\mathrm{HF}$ slides and $78 \%$ of EF slides reviewed by second reviewer showed better cytoplasmic staining. $\left(\mathrm{P}_{1}=0.084, \mathrm{P}_{2}=0.183\right)($ Table 2 , Table 3$)$

Out of 50 cases evaluated by first reviewer $90 \%$ of honey fixed (HF) slides and $96 \%$ of ethanol fixed (EF) showed preserved cell morphology, (Fig 2a \&2b) whereas $86 \%$ of $\mathrm{HF}$ slides and $78 \%$ of $\mathrm{EF}$ slides showed preserved cell morphology as reviewed by a second reviewer. $\left(\mathrm{P}_{1}=0.240, \mathrm{P}_{2}=0.183\right)$. (Table 2, Table 3)

On analysis of clarity of staining evaluated by first reviewer $98 \%$ of honey fixed (HF) slides and $96 \%$ of ethanol fixed (EF) (Fig 3a \&3b) showed acceptable clarity of staining, whereas $96 \%$ of HF slides and $74 \%$ of EF slides showed acceptable nuclear staining when reviewed by a second reviewer $\left(\mathrm{P}_{1}=0.558, \mathrm{P}_{2}=0.074\right)$. (Table 2, Table 3)

Out of 50 cases evaluated by first reviewer $92 \%$ of honey fixed (HF) slides and 90\% of ethanol fixed (EF) showed uniformity of staining, whereas $90 \%$ of $\mathrm{HF}$ slides and $76 \%$ of EF slides showed acceptable nuclear staining by a second reviewer showed acceptable uniformity of staining $\left(\mathrm{P}_{1}=0.727, \mathrm{P}_{2}=0.118\right)$, when compared with ethanol fixed slides with no statistical significance between them. (Table 2, Table 3)

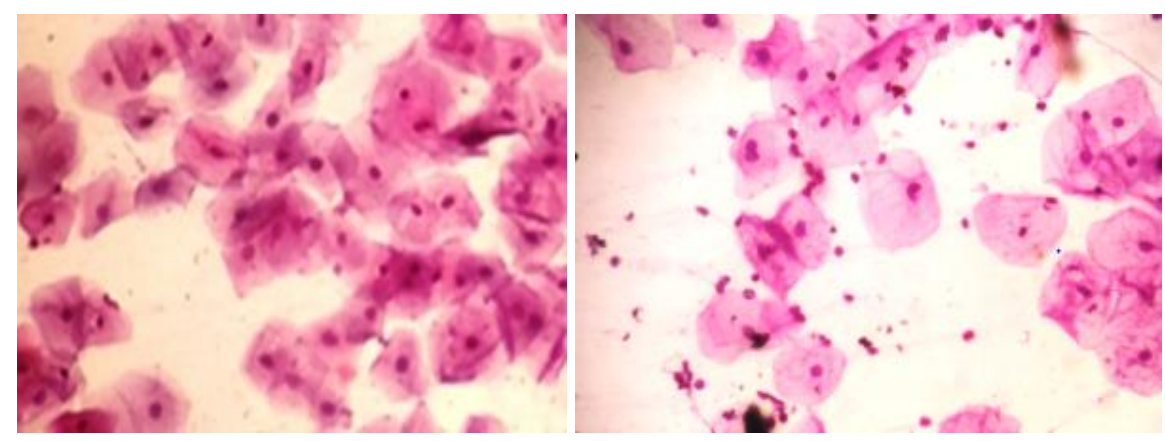

Fig. 1: 1a \&1b (400X): Acceptable nuclear and cytoplasmic staining of HF and EF smears respectively

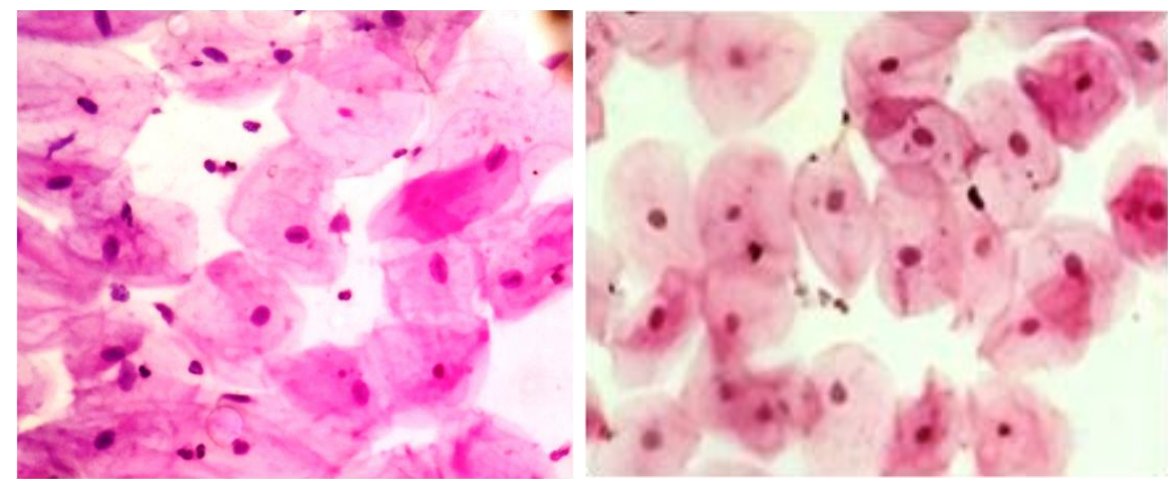

Fig. 2a \&2b: (400X): Acceptable uniformity and clarity of staining of HF and EF smears respectively
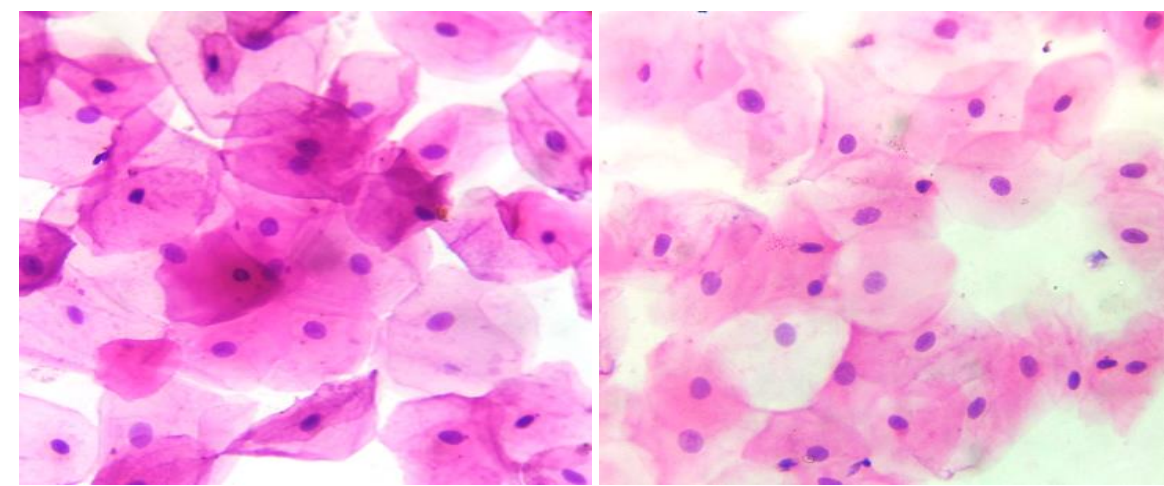

Fig. 3a \&3b (400X): Well preserved cell morphology of HF and EF smears respectively 
Table 1: Evaluation criteria

\begin{tabular}{|c|c|c|}
\hline Features & Scores and criteria & Scores and criteria \\
\hline Nuclear staining & $\begin{array}{c}\text { Acceptable }=1 \\
\text { Round, smooth and clear nuclear } \\
\text { membrane }\end{array}$ & $\begin{array}{c}\text { Unacceptable }=0 \\
\text { Granular, disintegrated and out of } \\
\text { focus }\end{array}$ \\
\hline Cytoplasmic staining & $\begin{array}{c}\text { Acceptable }=1 \\
\text { Intracytoplasmic membrane and } \\
\text { transparent cytoplasm }\end{array}$ & $\begin{array}{c}\text { Unacceptable }=0 \\
\text { Disintegrated cytoplasmic } \\
\text { membrane, granular cytoplasm and } \\
\text { out of focus } \\
\end{array}$ \\
\hline Cell morphology & $\begin{array}{c}\text { Preserved }=1 \\
\text { Absence of folds, no overlap and } \\
\text { maintained nuclear to cytoplasmic ratio }\end{array}$ & $\begin{array}{c}\text { Unpreserved }=0 \\
\text { Over lapping cells, folded and } \\
\text { disintegrated cells }\end{array}$ \\
\hline Clarity of staining & $\begin{array}{c}\text { Present }=1 \\
\text { Crispness in staining and transparency }\end{array}$ & $\begin{array}{c}\text { Absent }=0 \\
\begin{array}{c}\text { Obliterate the nucleus and } \\
\text { cytoplasm }\end{array}\end{array}$ \\
\hline Uniformity of staining & $\begin{array}{c}\text { Present }=1 \\
\begin{array}{c}\text { Uniformly stained throughout the } \\
\text { individual cell }\end{array}\end{array}$ & $\begin{array}{c}\text { Absent }=0 \\
\text { Stained in different shades of color } \\
\text { in an individual cell }\end{array}$ \\
\hline
\end{tabular}

Table 2: Reviewer 1

\begin{tabular}{|l|c|c|c|c|c|}
\hline \multirow{2}{*}{\multicolumn{1}{|c|}{ Staining }} & \multicolumn{2}{|c|}{ Ethanol } & \multicolumn{2}{c|}{ Honey } & \multirow{2}{*}{ p value } \\
\cline { 2 - 5 } & $\mathbf{N}$ & $\mathbf{\%}$ & $\mathbf{N}$ & $\mathbf{\%}$ & \\
\hline Nuclear staining & 49 & $98 \%$ & 50 & $100 \%$ & 0.315 \\
\hline Cytoplasmic staining & 46 & $92 \%$ & 40 & $80 \%$ & 0.084 \\
\hline Cell morphology & 48 & $96 \%$ & 45 & $90 \%$ & 0.240 \\
\hline Clarity of staining & 48 & $96 \%$ & 49 & $98 \%$ & 0.558 \\
\hline Uniformity of staining & 45 & $90 \%$ & 46 & $92 \%$ & 0.727 \\
\hline
\end{tabular}

Table 3: Reviewer 2

\begin{tabular}{|l|c|c|c|c|c|}
\hline \multirow{2}{*}{ Staining } & \multicolumn{2}{|c|}{ Ethanol } & \multicolumn{2}{c|}{ Honey } & \multirow{2}{*}{ p value } \\
\cline { 2 - 4 } & N & \% & N & \% & \\
\hline Nuclear staining & 38 & $76 \%$ & 44 & $92 \%$ & 0.118 \\
\hline Cytoplasmic staining & 39 & $78 \%$ & 44 & $88 \%$ & 0.183 \\
\hline Cell morphology & 39 & $78 \%$ & 44 & $86 \%$ & 0.183 \\
\hline Clarity of staining & 37 & $74 \%$ & 44 & $96 \%$ & 0.074 \\
\hline Uniformity of staining & 38 & $76 \%$ & 44 & $90 \%$ & 0.118 \\
\hline
\end{tabular}

\section{Discussion}

Group of cells which are building blocks of living organisms unite to form a tissue which perform specific function. Microscopic study of individual cell in a smear is called cytology and study of tissue is called histology. For appropriate cytological evaluation proper collection, fixation, staining and evaluation are required. Each of these steps plays a vital role in cytological diagnosis.

Ethanol is a well known and widely accepted fixative in cytopathology providing excellent preservation of morphology and cellular details which are the basic requirement to make cytological diagnosis. Ethanol being an alcohol fixative preserves the tissue antigens and decreases the turnaround time and cost which are required during antigen retrieval. ${ }^{5}$

Ethanol though an efficient cytological fixative has few disadvantages such as it is subjected to pilferage, expensive, flammable, evaporates easily and not freely available. It usually causes skin and eye irritation. License is required to obtain ethanol for its use in laboratory. This is one of the major limitations of ethanol being not freely available. ${ }^{6}$

In search of eco-friendly and ideal fixative many natural sweeteners are being experimented, among which honey has given promising results. Many studies have proved its efficacy in histopathology. Considering its fixation ability in histopathology it has been experimented in few cytological studies. Use of honey in funerary practices in many different cultures is well documented. A custom of preserving chief abbots in coffins full of honey by Burmese priests and mummification in honey by Egyptians is very well known. ${ }^{7}$

Honey is well known natural reliable sweetener. It is produced from many floral sources and contains carbohydrates, vitamins, minerals, and several trace elements. Honey has inherent antibacterial, anti- 
oxidative properties due to high osmolarity, low $\mathrm{pH}$ and the presence of components such as ascorbic acid, hydrogen peroxide and phenol inhibine. ${ }^{7}$

Honey preserves the tissue morphology similar to formalin in histopathology and has been experimented widely. Probable mechanism of fixation is presence of fructose which causes breakdown of aldehyde. These aldehydes then cross-link with tissue amino acids which leads to tissue fixation. ${ }^{8}$ Hence, considering this honey has also been experimented as fixative in cytology which has provided excellent cellular preservation and dehydration which are required for fixing the smears in cytopathology.

In honey fixed smears one could very clearly appreciate all cellular details such as nuclear staining, cytoplasmic staining, cellular morphology, clarity and uniformity of the staining which are almost equivalent to ethanol fixed smears.

In present study out of 50 cases evaluated by first reviewer $100 \%$ of honey fixed (HF) slides and $98 \%$ of ethanol fixed (EF) showed better nuclear staining, where as $92 \%$ of $\mathrm{HF}$ slides and $76 \%$ of EF slides showed acceptable nuclear staining by second reviewer $\left(\mathrm{P}_{1}=0.315, \mathrm{P}_{2}=0.118\right) .\left(\mathrm{P}_{1}=\mathrm{P}\right.$-value, first reviewer, $\mathrm{P}_{2}=\mathrm{P}$ value, second reviewer) (Table 2, Table 3 )

Similarly out of 50 cases evaluated by first reviewer $80 \%$ of honey fixed (HF) slides and $92 \%$ of ethanol fixed (EF) showed better cytoplasmic staining, where as $88 \%$ of $\mathrm{HF}$ slides and $78 \%$ of EF slides showed acceptable nuclear staining by second reviewer showed better cytoplasmic staining. $\left(\mathrm{P}_{1}=0.084, \mathrm{P}_{2}=\right.$ 0.183) (Table 2, Table 3)

In present study out of 50 cases evaluated by first reviewer $90 \%$ of honey fixed (HF) slides and $96 \%$ of ethanol fixed (EF) showed preserved cell morphology, where as $86 \%$ of $\mathrm{HF}$ slides and $78 \%$ of $\mathrm{EF}$ slides showed acceptable nuclear staining by second reviewer showed preserved cell morphology. $\left(\mathrm{P}_{1}=0.240, \mathrm{P}_{2}=\right.$ 0.183 ).

Out of 50 cases evaluated by first reviewer $98 \%$ of honey fixed (HF) slides and $96 \%$ of ethanol fixed (EF) showed acceptable clarity of staining, whereas $96 \%$ of HF slides and $74 \%$ of EF slides showed acceptable nuclear staining by a second reviewer showed acceptable clarity of staining $\left(\mathrm{P}_{1}=0.558, \mathrm{P}_{2}=0.074\right)$.

Out of 50 cases evaluated by first reviewer $92 \%$ of honey fixed (HF) slides and 90\% of ethanol fixed (EF) showed uniformity of staining, whereas $90 \%$ of HF slides and $76 \%$ of EF slides showed acceptable nuclear staining by a second reviewer showed acceptable uniformity of staining $\left(\mathrm{P}_{1}=0.727, \mathrm{P}_{2}=0.118\right)$, when compared with ethanol fixed slides with no statistical significance between them. In the present study all cellular parameters were in concordance with Singh et al study.

Immunohistochemical comparison done in Ozakan $\mathrm{N}$ et $\mathrm{al}^{7}$ study for honey fixed and formalin fixed paraffin embedded tissue with Vimentin and Ki67 showed convincing results. There were no statistically significant differences among the three fixatives concerning the expressions of vimentin and Ki-67 (p > 0.05). ${ }^{9}, 10$

Honey due to its inherent antimicrobial and fixative property can be used as natural and cheaper alternative to ethanol as fixative. It can also be exploited in camps and places where ethanol is not freely available. Further establishment of its nature and outcome in Immunohistochemistry with various other antigens are required.

\section{References}

1. Jindal S, Chauhan I, Grewal HK. Alteration in buccal mucosal cells due to the effect of tobacco and alcohol by assessing the silver-stained nucleolar organiser regions and micronuclei. Journal of Cytology. 2013;30(3):174.

2. Sloan P, Bocking A. Oral cavity. In Gray W, Kocjan G. Diagnostic Cytopathology E-Book: Expert Consult: Online and Print. Elsevier Health Sciences; 2010;24:2534.

3. McCarthy J. antibacterial effects of honey: medical fact or fiction?. American Bee Journal. 1995:171-2.

4. Molan PC. Why honey is effective as a medicine. 1. Its use in modern medicine. Bee world. 1999;80(2):80-92.

5. Singh A, Hunasgi S, Koneru A, Vanishree M, Ramalu S, Manvikar V. Comparison of honey with ethanol as an oral cytological fixative: A pilot study. Journal of cytology. 2015;32(2):113.

6. Ishaq R, Azmat H, Omair M, Sheikh AK, Tanwani AK. Comparison of Honey With Alcohol As A Fixative In Fine Needle Aspiration Cytology. International Journal of Pathology. 2017;15(1):15-8.

7. Özkan N, Şalva E, Cakalağaoğlu F, Tüzüner B. Honey as a substitute for formalin?. Biotechnic \& Histochemistry. 2012;87(2):148-53.

8. Patil S, Rao RS, Ganavi BS, Majumdar B. Natural sweeteners as fixatives in histopathology: A longitudinal study. Journal of natural science, biology, and medicine. 2015;6(1):67.

9. Majumdar B, Rao R, Patil S. Tissue Preservation with Natural Fixatives: An Immunohistochemical Evaluation. World Journal of Dentistry. 2016;7:87-91.

10. Gunter M, Bryant P. Immunocytochemical evaluation of ductal carcinoma in breast after preservation in honey. Journal of Histotechnology. 2009;32(2):54-9.

How to cite this article: Nerune S.M, Khan M.N,
Potekar R.M, Patil V. Natural versus synthetic
fixative in oral cytological smears - A double
blind study. Indian J Pathol Oncol.
2018;5(4):663-666.

